\title{
Economic pricing techniques for transmission network in deregulated electricity market
}

\author{
Shaikashraf ali ${ }^{1}$ K.Vimala kumar ${ }^{2}$ \\ P.G.Scholor, E.E.E Department, J.N.T.U.A College of Engineering,Pulivendula,Kadappa,India ${ }^{1}$ \\ Assistant professor, E.E.E Department, J.N.T.U.A College of Engineering,Pulivendula,Kadappa,India ${ }^{2}$
}

\begin{abstract}
The aim of deregulation is to introduce an element of competition into electrical energy delivery and thereby allow market forces to price energy at low rates for the customer and higher efficiency for the suppliers. The necessity for deregulation is to provide cheaper electricity, to offer greater choice to the customer in purchasing the economic energy, to give more choice of generation and to offer better services with respect to power quality i.e. constant voltage, constant frequency and uninterrupted power supplythis paper provides a methodology to apportion the cost of the transmission network to generators and demands that use it. How to allocate the cost of the transmission network is an open research issue as available techniques embody important simplifying assumptions, which may render controversial results. In this paper three techniques namely $Z_{\text {bus }}$ method, $Z_{b u s}$ avgmethod and Relative Electrical Distance (RED) method for the network cost allocation is compared. It has been successfully applied on an IEEE 24 bus-Reliability Test System (RTS) and the results obtained are compared.
\end{abstract}

Keywords: Transmission network cost allocation, active power flow, generator cost contribution, load cost contribution, $\mathrm{Z}_{\text {bus }}, \mathrm{Z}_{\text {bus }}$ avg and RED.

\section{INTRODUCTION}

Deregulation word refers to un-bundling of electrical Consider the complex power flow $S_{J K}$ computed at bus $j$ utility or restructuring of electrical utility and allowing and flowing through the line connecting bus $j$ to bus $k$ private companies to participate. The aim of deregulation asshown in Figure 1.As the power flow solution is known, is to introduce an element of competition into electrical weselect the direction of the complex power flow so that energy delivery and thereby allow market forces to price $P_{J K}>0$

energy at low rates for the customer and higher efficiency for the suppliers. In the traditional pro rata method [1], [2] The complex power flow $S_{\mathrm{jk}}$ is

bothgenerators and loads are charged a flat rate per $S_{j k}=V_{J} I_{j k}^{*}$ megawathour, disregarding their respective use of individual transmission lines. Flow-based method [3] estimates the usage of the lines by generators and demands and charges them accordingly. Some flow-based methods use theproportional sharing principle [4], [5], which implies thatany active power flow leaving a bus is proportionally madeup of the flows entering that bus, such that Kirchhoff'sCurrent Law is satisfied. Other methods that use generationshift distribution factors [6] are dependent on the selectionof the slack bus and lead to controversial results. The usagebasedmethod reported in [7] and [8] uses the so-calledequivalent bilateral exchanges (EBEs).

\section{PROBLEM STATEMENT}

A. Background of $Z_{\text {bus }}$ and $Z_{\text {bus }}$ avg technique

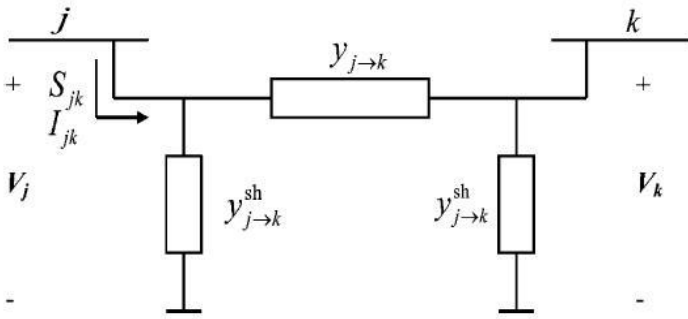

Figure 1. П equivalent circuit of line section $j k$
This complexpower flow equation can be written as

$$
\begin{aligned}
& \mathrm{S}_{\mathrm{jk}}=\mathrm{V}_{\mathrm{j}} \sum_{\mathrm{i}=1}^{\mathrm{n}}\left(\mathrm{a}_{\mathrm{jk}}^{\mathrm{i}} \mathrm{I}_{\mathrm{i}}\right)^{*}=\sum_{\mathrm{i}=1}^{\mathrm{n}} \mathrm{V}_{\mathrm{j}}\left(\mathrm{a}_{\mathrm{jk}}^{\mathrm{i}^{*}} \mathrm{I}_{\mathrm{i}}^{*}\right) \\
& \text { Here } \mathrm{a}_{\mathrm{jk}}^{\mathrm{i}}=\left(\mathrm{Z}_{\mathrm{ji}}-\mathrm{Z}_{\mathrm{ki}}\right) \mathrm{Y}_{\mathrm{jk}}+\mathrm{Z}_{\mathrm{ji}} \mathrm{Y}_{\mathrm{jk}}^{\text {sh }}
\end{aligned}
$$

We know that the power flow through any line is

$$
\mathrm{P}_{\mathrm{jk}}^{\mathrm{i}}=\operatorname{Real}\left\{\mathrm{V}_{\mathrm{j}} \mathrm{a}_{\mathrm{jk}}^{\mathrm{i}_{\mathrm{i}}^{*}} \mathrm{I}_{\mathrm{i}}^{*}\right\}
$$

\section{B. Transmission cost allocation using $Z_{\text {bus }}$}

$$
\mathrm{U}_{\mathrm{jk}}^{\mathrm{i}}=\left|\mathrm{P}_{\mathrm{jk}}^{\mathrm{i}}\right|
$$

Total usage of the line $\mathrm{jk}$ is

$$
\mathrm{U}_{\mathrm{jk}}=\sum_{\mathrm{i}=1}^{\mathrm{n}} \mathrm{U}_{\mathrm{jk}}^{\mathrm{i}}
$$

If bus i contains only generation, the usage allocated to generation i pertaining to line $\mathrm{jkis}$

$$
\mathrm{U}_{\mathrm{jk}}^{\mathrm{Gi}}=\mathrm{U}_{\mathrm{jk}}^{\mathrm{i}}
$$

If bus $\mathrm{i}$ contains only demand, the usage allocated to demandi pertaining to line jkis

$$
U_{j \mathrm{k}}^{\text {Di }}=U_{j \mathrm{k}}^{\mathrm{i}}
$$

For the sake of simplicity and for each line, total annualized line cost in $\$ / \mathrm{h}, \mathrm{C} \mathrm{jk}$, which includes operation , maintenance and building costs is considered. The corresponding cost rate for line jkis then

$$
\mathrm{r}_{\mathrm{jk}}=\mathrm{C}_{\mathrm{jk}} / \mathrm{U}_{\mathrm{jk}}
$$

In this way, the cost of line jkallocated to the generator located at bus $i$ is

$$
\mathrm{C}_{\mathrm{jk}}^{\mathrm{Gi}}=\mathrm{r}_{\mathrm{jk}} \mathrm{U}_{\mathrm{jk}}^{\mathrm{Gi}}
$$


Similarly, cost of line jkallocated to the demand located at The elements of $\left[\mathrm{F}_{\mathrm{LG}}\right]$ matrix are complex. Its columns bus $i$ is

$$
C_{j k}^{D i}=r_{j k} U_{j k}^{D i}
$$

Finally, the total transmission cost of the network the generator located at a bus $\mathrm{i}$ is

$$
C^{G i}=\sum_{(j, k) \in \Omega_{L}} r_{j k} U_{j k}^{G i}(11)
$$

Similarly, cost of line jkallocated to the demand located at bus $i$ is

$$
C^{D i}=\sum_{(j, k) \in \Omega_{L}} r_{j k} U_{j k}^{D i}
$$

correspond to the generator bus numbers and rows correspond to the load bus numbers. This matrix gives the relation between load bus and source bus voltages. Ideal generation proportions are obtained from $a b s\left[F_{L G}\right]$ matrix, also known as desired generation proportions matrix $\left[\mathrm{D}_{\mathrm{LG}}\right]$ as

$$
\left[\mathrm{D}_{\mathrm{LG}}\right]=\operatorname{abs}\left\{\left[\mathrm{F}_{\mathrm{LG}}\right]\right\}
$$

$D_{L G}$ ] gives the information about the location of load nodes with respect to generator nodes, which is

Equation (2) is written in such a manner that $\mathrm{P}_{\mathrm{jk}} \geq 0$, that is, in the direction of the active power flows. However, (2) can also be written in the direction of the active power counter-flows, which leads to distance parameters $\mathrm{a}_{\mathrm{jk}}^{\mathrm{i}}$. It is correct to write Equation (2) in both the ways. However, (3) shows that distance parameters are not generally symmetrical with respect to line indexes, i.e., $a_{j k}^{i} \neq$ $a_{k j}^{i}$, which results in different usage allocations depending on whether (2) is written in the direction of the active powerflows or counter-flows.

Now, to address these two types of power flows, two $Z_{\text {bus }}$ based techniques are used. The first one is denoted by $Z_{\text {bus }}$ and is based on (2) written in the direction of the active power flows. This is a common way as the actual activepower flows directions are used. This selection generallyresults in higher usage allocation to generators versusdemands. The second technique denoted by $Z_{\text {bus }}^{\text {avg }}$ provide the average value of allocated cost (usage) using the $Z_{\text {bus }}$ technique with (2) written in the direction of the activepower counter-flows. This technique smoothens the trend ofallocating higher network usage to generators versusdemands.

\section{Background of RED technique}

Consider a system where $\mathrm{n}$ is the total number of buses with $1,2 \ldots \mathrm{g}$, where $\mathrm{g}$ is the number of generator buses and $\mathrm{g}+1, \ldots, \mathrm{n}$, remaining $(\mathrm{n}-\mathrm{g})$ are the load buses. For a given system, the network admittance matrix is given by

$\left[\begin{array}{c}I_{G} \\ I_{L}\end{array}\right]=\left[\begin{array}{ll}Y_{G G} & Y_{G L} \\ Y_{L G} & Y_{L L}\end{array}\right]\left[\begin{array}{c}V_{G} \\ V_{L}\end{array}\right]$

popularly termed as RED. The [RED] is obtained from the $\left[D_{L G}\right]$ matrix as

$$
[\mathrm{RED}]=\mathrm{M}-\left[\mathrm{D}_{\mathrm{LG}}\right]
$$

Where, $M$ is the unity matrix of size $L X G, G$ is the number of generator buses and $\mathrm{L}$ is the number of load buses.

\section{Evaluation of the power contract transmission matrix and transmission cost matrix}

Evaluation of the power contract transmission matrix and transmission cost matrixThe power contract transmission matrix $\left[\mathrm{P}_{\mathrm{LG}}\right]$ is calculated from the transaction details between the generatorand the load from which $\left[\mathrm{C}_{\mathrm{LG}}\right]$ transmission cost matrix iscalculated using the following expression

$\left[\mathrm{C}_{\mathrm{LG}}\right]=\{\mathrm{X}+[\mathrm{RED}]\}$

where the transmission charges are directly proportional to the relative electrical distances and it is assumed that the charges for the consumers are Rsx. The transmission charges are calculated by each element of $\left[\mathrm{C}_{\mathrm{LG}}\right]$ matrix multiplied by the corresponding element of $\left[\mathrm{P}_{\mathrm{LG}}\right]$ matrix.

\section{III.IMPLEMENTATION AND RESULT}

All the three methodologies are compared by testing it on a standard IEEE 24 bus reliability test system shown in fig.2.

\section{A.Z-bus Technique}

Where $\mathrm{I}_{\mathrm{G}}, \mathrm{I}_{\mathrm{L}}$ and $\mathrm{V}_{\mathrm{G}}, \mathrm{V}_{\mathrm{L}}$ represent complex current and voltage vectors at the generators and load nodes.

$\left[\mathrm{Y}_{\mathrm{GG}}\right],\left[\mathrm{Y}_{\mathrm{GL}}\right],\left[\mathrm{Y}_{\mathrm{LG}}\right]$ and $\left[\mathrm{Y}_{\mathrm{LL}}\right]$ are corresponding portions of network Y-bus matrix

$\left[\mathrm{I}_{\mathrm{G}}\right]=\left[\mathrm{Y}_{\mathrm{GG}}\right]\left[\mathrm{V}_{\mathrm{G}}\right]+\left[\mathrm{Y}_{\mathrm{GL}}\right]\left[\mathrm{V}_{\mathrm{L}}\right]$
$\left[\mathrm{I}_{\mathrm{L}}\right]=\left[\mathrm{Y}_{\mathrm{LG}}\right]\left[\mathrm{V}_{\mathrm{G}}\right]+\left[\mathrm{Y}_{\mathrm{LL}}\right]\left[\mathrm{V}_{\mathrm{L}}\right]$

$\left[\mathrm{I}_{\mathrm{L}}\right]=\left[\mathrm{Y}_{\mathrm{LG}}\right]\left[\mathrm{V}_{\mathrm{G}}\right]+\left[\mathrm{Y}_{\mathrm{LL}}\right]\left[\mathrm{V}_{\mathrm{L}}\right]$

$\left[\mathrm{V}_{\mathrm{L}}\right]=\left[\mathrm{Y}_{\mathrm{LL}}\right]^{-1}\left[\mathrm{I}_{\mathrm{L}}\right]-\left[\mathrm{Y}_{\mathrm{LL}}\right]^{-1}\left[\mathrm{Y}_{\mathrm{LG}}\right]\left[\mathrm{V}_{\mathrm{G}}\right]$

Substituting $\left[\mathrm{V}_{\mathrm{L}}\right]$ in (14), we obtain below equation no(17)

$$
\begin{gathered}
{\left[\mathrm{I}_{\mathrm{G}}\right]=\left[\mathrm{Y}_{\mathrm{GG}}\right]\left[\mathrm{V}_{\mathrm{G}}\right]+\left[\mathrm{Y}_{\mathrm{GL}}\right]\left\{\left[\mathrm{Y}_{\mathrm{LL}}\right]^{-1}\left[\mathrm{I}_{\mathrm{L}}\right]-\left[\mathrm{Y}_{\mathrm{LL}}\right]^{-1}\left[\mathrm{Y}_{\mathrm{LG}}\right]\left[\mathrm{V}_{\mathrm{G}}\right]\right\}} \\
\text { From the equations }(16) \text { and }(17) \text { can be written as } \\
{\left[\begin{array}{c}
\mathrm{V}_{\mathrm{L}} \\
\mathrm{I}_{\mathrm{G}}
\end{array}\right]=\left[\begin{array}{cc}
\mathrm{Z}_{\mathrm{LL}} & \mathrm{F}_{\mathrm{LG}} \\
\mathrm{K}_{\mathrm{GL}} & \mathrm{Y}_{\mathrm{GG}}^{\prime} \\
{\left[\mathrm{F}_{\mathrm{LG}}\right]=-\left[\mathrm{I}_{\mathrm{L}}\right.} \\
\left.\mathrm{I}_{\mathrm{G}}\right]
\end{array}\right.} \\
\text { Where }\left[\mathrm{K}_{\mathrm{LG}}\right]=\left[\mathrm{Y}_{\mathrm{GL}}\right]\left[\mathrm{Y}_{\mathrm{LL}}\right]^{-1}\left[\mathrm{Y}_{\mathrm{L}}\right] \\
{\left[\mathrm{Y}_{\mathrm{GG}}^{\prime}\right]=\left\{\left[\mathrm{Y}_{\mathrm{GG}}\right]-\left[\mathrm{Y}_{\mathrm{GL}}\right]\left[\mathrm{Y}_{\mathrm{LL}}\right]^{-1}\left[\mathrm{Y}_{\mathrm{LG}}\right]\right\}}
\end{gathered}
$$

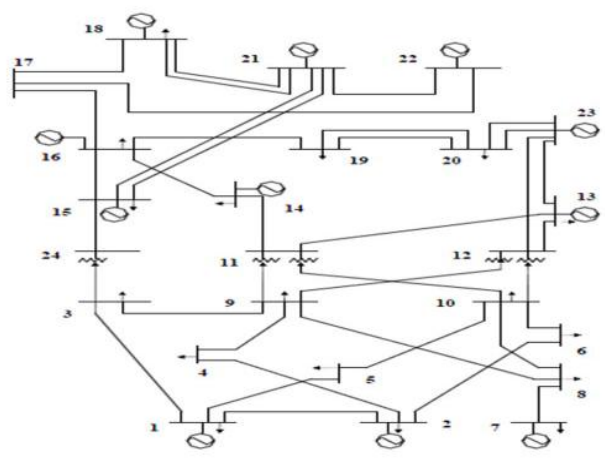

Fig. 2 RTS 24 Bus System 
INTERNATIONAL JOURNAL OF INNOVATIVE RESEARCH IN ELECTRICAL, ELECTRONICS, INSTRUMENTATION AND CONTROL ENGINEERING Vol. 2, Issue 12, December 2014

TABLE I. GENERATOR COST CONTRIBUTIONS $\mathrm{C}_{0}(\mathrm{k}, \mathrm{i})$ IN $\mathrm{P}_{\mathrm{j} \mathrm{k}}>0$ DIRECTION OF $\mathrm{Z}_{\text {bus }}$ TECHNIQUE

\begin{tabular}{|c|c|c|c|c|c|c|c|c|c|c|}
\hline $\begin{array}{l}\text { LINE/ } \\
\text { GEN }\end{array}$ & GEN1 & GEN2 & GEN7 & GEN13 & GEN15 & GEN16 & GEN18 & GEN21 & GEN22 & GEN23 \\
\hline 1 & 1.5454 & 2.1819 & 0.2081 & 0.0057 & 0.0435 & 0.0144 & 0.0387 & 0.3168 & 0.2019 & 0.04077 \\
\hline 2 & 5.9171 & 6.5095 & 0.9888 & 0.0195 & 5.0995 & 2.1063 & 1.3145 & 36.7163 & 22.8753 & 29.5241 \\
\hline 3 & 6.6457 & 7.2634 & 1.6037 & 0.6195 & 0.4694 & 0.1381 & 0.5175 & 1.3723 & 0.2573 & 7.2106 \\
\hline 4 & 3.4813 & 4.9031 & 2.6327 & 0.0743 & 2.1951 & 0.9758 & 0.3781 & 16.0133 & 9.9996 & 19.8214 \\
\hline 5 & 8.2860 & 11.2595 & 1.6975 & 0.1162 & 3.4800 & 1.4237 & 0.6828 & 24.8784 & 15.4713 & 22.5651 \\
\hline 6 & 0.9544 & 0.7765 & 4.1011 & 0.0834 & 2.4655 & 0.8803 & 1.0394 & 17.3398 & 10.7659 & 0.3210 \\
\hline 7 & 0.8687 & 0.9995 & 0.5212 & 0.0090 & 2.6626 & 1.0610 & 0.7559 & 19.0127 & 11.8204 & 12.0753 \\
\hline 8 & 2.7614 & 3.8892 & 2.0894 & 0.0575 & 1.7429 & 0.7742 & 0.2988 & 12.7110 & 7.9364 & 15.7257 \\
\hline 9 & 6.4536 & 7.0535 & 1.5576 & 0.6020 & 0.4557 & 0.1343 & 0.5029 & 1.3309 & 0.2485 & 7.0061 \\
\hline 10 & 2.8576 & 3.9557 & 0.0628 & 0.6793 & 0.9696 & 0.1263 & 0.4768 & 5.2477 & 2.7156 & 0.2940 \\
\hline 11 & 0.0706 & 0.0865 & 38.3888 & 0.0119 & 0.1036 & 0.0511 & 0.0344 & 0.7917 & 0.5057 & 0.9766 \\
\hline 12 & 0.3932 & 0.5306 & 20.4205 & 0.2973 & 2.2119 & 0.7839 & 0.0302 & 14.9544 & 8.9917 & 13.1907 \\
\hline 13 & 0.3006 & 0.4155 & 22.3983 & 0.3028 & 2.0220 & 0.6996 & 0.0116 & 13.5628 & 8.1199 & 11.9299 \\
\hline 14 & 0.7888 & 1.0481 & 2.2779 & 0.0687 & 1.5259 & 0.7630 & 0.2116 & 11.5114 & 7.2730 & 11.3855 \\
\hline 15 & 0.8877 & 1.1743 & 2.5061 & 0.0285 & 0.9746 & 0.3627 & 0.2935 & 6.4991 & 3.8306 & 21.4688 \\
\hline 16 & 0.0419 & 0.0455 & 0.0264 & 0.5086 & 1.8470 & 1.1043 & 1.0377 & 15.3081 & 10.1223 & 18.5739 \\
\hline 17 & 0.0259 & 0.0212 & 0.1090 & 0.5969 & 1.6513 & 0.9692 & 0.8957 & 13.5882 & 8.9576 & 23.4659 \\
\hline 18 & 0.4198 & 0.5192 & 0.7132 & 0.9166 & 0.2288 & 0.0405 & 0.0809 & 1.3178 & 0.7135 & 19.7509 \\
\hline 19 & 0.2216 & 0.2858 & 0.5258 & 0.0168 & 1.0984 & 0.6290 & 0.4895 & 8.8903 & 5.8136 & 4.4636 \\
\hline 20 & 0.4092 & 0.5158 & 0.8250 & 0.7974 & 0.8784 & 0.4900 & 0.3364 & 7.0215 & 4.5618 & 9.4417 \\
\hline 21 & 0.6633 & 0.8381 & 1.3560 & 0.0826 & 1.5519 & 0.9090 & 0.7091 & 12.6715 & 8.3125 & 34.9946 \\
\hline 22 & 0.5332 & 0.6741 & 1.0932 & 0.4059 & 1.2638 & 0.7565 & 0.6190 & 10.4177 & 6.8629 & 35.2611 \\
\hline 23 & 0.1681 & 0.2193 & 0.4308 & 0.0116 & 1.0917 & 0.6190 & 0.4759 & 8.8006 & 5.7453 & 4.6935 \\
\hline 24 & 0.0271 & 0.0230 & 0.0871 & 0.0185 & 1.0912 & 0.1880 & 0.1581 & 4.6432 & 2.0891 & 2.6753 \\
\hline 25 & 0.1685 & 0.2044 & 0.2362 & 0.0159 & 0.4898 & 0.0065 & 0.4123 & 11.2728 & 5.8982 & 0.6095 \\
\hline 26 & 0.1685 & 0.2044 & 0.2362 & 0.0159 & 0.4898 & 0.0065 & 0.4123 & 11.2728 & 5.8982 & 0.6095 \\
\hline 27 & 0.6933 & 0.8114 & 0.5960 & 0.0009 & 1.4875 & 0.5895 & 0.4661 & 10.6385 & 6.6243 & 5.8392 \\
\hline 28 & 0.1337 & 0.1687 & 0.2698 & 0.0267 & 0.2807 & 0.1182 & 0.5518 & 8.6076 & 7.0481 & 2.3139 \\
\hline 29 & 0.0006 & 0.0048 & 0.0696 & 0.0309 & 0.5739 & 0.3097 & 0.2192 & 4.5336 & 2.9340 & 6.6243 \\
\hline 30 & 0.0224 & 0.0334 & 0.0812 & 0.0105 & 0.3094 & 0.0693 & 0.5156 & 7.6331 & 1.3438 & 1.1311 \\
\hline 31 & 0.8240 & 1.0214 & 1.4277 & 0.1284 & 0.1682 & 0.3781 & 0.1676 & 8.5001 & 57.1858 & 9.0696 \\
\hline 32 & 0.0009 & 0.0020 & 0.0374 & 0.0068 & 0.3155 & 0.0579 & 0.4214 & 7.3918 & 1.3492 & 0.8553 \\
\hline 33 & 0.0009 & 0.0020 & 0.0374 & 0.0068 & 0.3155 & 0.0579 & 0.4214 & 7.3918 & 1.3492 & 0.8553 \\
\hline 34 & 0.0091 & 0.0160 & 0.0763 & 0.0279 & 0.4900 & 0.2658 & 0.1898 & 3.8793 & 2.5129 & 5.8397 \\
\hline 35 & 0.0091 & 0.0160 & 0.0763 & 0.0279 & 0.4900 & 0.2658 & 0.1898 & 3.8793 & 2.5129 & 5.8397 \\
\hline 36 & 0.0229 & 0.0308 & 0.0703 & 0.0164 & 0.2434 & 0.1344 & 0.0984 & 1.9403 & 1.2606 & 3.2450 \\
\hline 37 & 0.0229 & 0.0308 & 0.0703 & 0.0164 & 0.2434 & 0.1344 & 0.0984 & 1.9403 & 1.2606 & 3.2450 \\
\hline 38 & 0.4123 & 0.5059 & 0.6490 & 0.0535 & 0.6160 & 0.0915 & 0.0039 & 8.1982 & 42.1776 & 3.0886 \\
\hline
\end{tabular}

Using Equation No 2, the cost of each line allocated to the load at various buses is computed .B.Z $Z_{\text {bus }}^{\text {avg Method }}$

The cost of each line allocated to the load and the generator located at various buses are calculated as per the discussion made earlier for $Z_{\text {bus }}^{\text {avg }}$ method

TABLE II. COMPARISON OF BOTH $Z_{b u s}$ AND $Z_{b u s}^{a v g}$ TECHNIQUES

\begin{tabular}{|l|l|l|l|l|l|l|}
\hline & \multicolumn{3}{|l|}{$\boldsymbol{Z}_{\text {bus }}$ Technique } & \multicolumn{2}{l|}{$\boldsymbol{Z}_{\text {bus }}^{\text {avg }}$ Technique } \\
Bus No. & $\begin{array}{c}\text { CG } \\
\text { in \$ }\end{array}$ & $\begin{array}{c}\text { CD } \\
\text { in \$ }\end{array}$ & $\begin{array}{c}\text { TOTAL COST } \\
\text { In \$ }\end{array}$ & $\begin{array}{l}\mathbf{C G}^{\text {avg }} \\
\text { In \$ }\end{array}$ & $\begin{array}{l}\mathbf{C D}^{\text {avg }} \\
\text { In \$ }\end{array}$ & $\begin{array}{c}\text { TOTAL COST }^{\text {avg }} \\
\text { In \$ }\end{array}$ \\
\hline 1. & 47.2130 & 29.6454 & 76.8584 & 46.1091 & 28.9522 & 75.0613 \\
\hline 2. & 58.2409 & 32.8452 & 91.0861 & 56.1646 & 31.6742 & 87.8389 \\
\hline 3. & 0 & 182.7847 & 182.7847 & 0 & 193.0269 & 193.0269 \\
\hline 4. & 0 & 83.9559 & 83.9559 & 0 & 83.1586 & 83.1586 \\
\hline 5. & 0 & 67.6732 & 67.6732 & 0 & 68.2859 & 68.2859 \\
\hline 6. & 0 & 61.5377 & 61.5377 & 0 & 52.9137 & 52.9137 \\
\hline 7. & 110.551 & 57.5808 & 168.1359 & 112.6909 & 58.6932 & 171.3841 \\
\hline 8. & 0 & 163.0228 & 163.0228 & 0 & 169.1423 & 169.1423 \\
\hline 9. & 0 & 141.7184 & 141.7184 & 0 & 147.7805 & 147.7805 \\
\hline 10. & 0 & 76.4259 & 76.4259 & 0 & 80.6095 & 80.6095 \\
\hline 11. & 0 & 0 & 0 & 0 & 0 & 0 \\
\hline 12. & 0 & 0 & 0 & 0 & 0 & 0 \\
\hline 13. & 6.7154 & 9.5297 & 16.2451 & 7.4862 & 10.6236 & 18.1098 \\
\hline
\end{tabular}


INTERNATIONAL JOURNAL OF INNOVATIVE RESEARCH IN ELECTRICAL, ELECTRONICS, INSTRUMENTATION AND CONTROL ENGINEERING Vol. 2, Issue 12, December 2014

\begin{tabular}{|l|l|l|l|l|l|l|}
\hline 14. & 0 & 113.2145 & 113.2145 & 0 & 128.6278 & 128.6278 \\
\hline 15. & 43.6373 & 64.3396 & 107.9768 & 48.4550 & 71.4429 & 119.8979 \\
\hline 16. & 18.4858 & 11.9263 & 30.4121 & 21.2159 & 13.6877 & 34.9036 \\
\hline 17. & 0 & 0 & 0 & 0 & 0 & 0 \\
\hline 18. & 15.5568 & 12.9510 & 28.5078 & 17.9632 & 14.9543 & 32.9175 \\
\hline 19. & 0 & 122.1187 & 122.1187 & 0 & 138.0465 & 138.0465 \\
\hline 20. & 0 & 53.3961 & 53.3961 & 0 & 61.4568 & 61.4568 \\
\hline 21. & 361.9979 & 0 & 361.9979 & 402.1204 & 0 & 402.1204 \\
\hline 22. & 303.4934 & 0 & 303.4934 & 326.7817 & 0 & 326.7817 \\
\hline 23. & 376.6848 & 0 & 376.6848 & 427.7452 & 0 & 427.7452 \\
\hline 24. & 0 & 0 & 0 & 0 & 0 & 0 \\
\hline
\end{tabular}

The above table gives the information about the cost allocated to different generators and loads for IEEE RTS 24bus system for the $Z_{\text {bus }}$ based techniques. Though $Z_{\text {bus }}$ the methods yield the same total transmission cost i.e TOTAL COST = $\$ 2627.246$, it is inferred that the $Z_{\text {bus }}$ technique allocates more usage to generators rather than

Demands and similarly allocates most of the cost to generators compared to demands. The $Z_{b u s}^{a v g}$ technique avoids the allocating most of the cost to generators than demands.

\section{D.RED method.}

Using Equation No 28, the desired load sharing/generation scheduling for the standard IEEE 24 bus RTS is calculated and is shown in Table III. All schedules are shown in MW with an assumption of same load of 250MW at each load bus.

\begin{tabular}{|c|c|c|c|c|c|c|c|c|c|c|c|}
\hline Load Bus & \multicolumn{9}{|c|}{ Power drawn from each Generator } & Total \\
\hline No & G1 & G2 & G7 & G13 & G15 & G16 & G18 & G21 & G22 & G23 & $\begin{array}{c}\text { Load } \\
(\text { MW) }\end{array}$ \\
\hline 1 & 163.625 & 86.375 & 0 & 0 & 0 & 0 & 0 & 0 & 0 & 0 & 250 \\
\hline 2 & 0 & 93.25 & 0 & 156.75 & 0 & 0 & 0 & 0 & 0 & 0 & 250 \\
\hline 3 & 0 & 111.55 & 0 & 0 & 0 & 138.4495 & 0 & 0 & 0 & 0 & 250 \\
\hline 4 & 0 & 87.775 & 0 & 0 & 162.225 & 0 & 0 & 0 & 0 & 0 & 250 \\
\hline 5 & 0 & 58.35 & 0 & 0 & 0 & 0 & 191.65 & 0 & 0 & 0 & 250 \\
\hline 6 & 0 & 126.675 & 0 & 0 & 0 & 0 & 0 & 123.325 & 0 & 0 & 250 \\
\hline 7 & 149.725 & 0 & 100.275 & 0 & 0 & 0 & 0 & 0 & 0 & 0 & 250 \\
\hline 8 & 0 & 149.725 & 65.825 & 0 & 0 & 0 & 34.4502 & 0 & 0 & 0 & 250 \\
\hline 9 & 0 & 88.775 & 0 & 0 & 0 & 161.225 & 0 & 0 & 0 & 0 & 250 \\
\hline 10 & 0 & 0 & 0 & 0 & 0 & 0 & 39.3497 & 210.6197 & 0 & 0 & 250 \\
\hline 13 & 0 & 0 & 0 & 196.125 & 0 & 0 & 0 & 53.875 & 0 & 0 & 250 \\
\hline 14 & 0 & 0 & 0 & 0 & 158.375 & 0 & 0 & 0 & 0 & 91.625 & 250 \\
\hline 15 & 0 & 0 & 0 & 0 & 0 & 108.725 & 0 & 0 & 141.275 & 0 & 250 \\
\hline 16 & 0 & 0 & 0 & 0 & 68.475 & 58.525 & 0 & 0 & 0 & 123 & 250 \\
\hline 18 & 0 & 110.85 & 0 & 0 & 28.9499 & 0 & 0 & 0 & 0 & 110.1998 & 250 \\
\hline 19 & 0 & 0 & 0 & 85.375 & 0 & 0 & 164.6235 & 0 & 0 & 0 & 250 \\
\hline 20 & 0 & 0 & 0 & 99.7245 & 0 & 0 & 0 & 0 & 150.275 & 0 & 250 \\
\hline Total & 313.35 & 913.325 & 166.1 & 537.9729 & 418.0244 & 466.925 & 464.5232 & 387.8192 & 291.55 & 324.825 & 4250 \\
\hline
\end{tabular}

\section{E. Evaluation of Transmission Charges}

Total Generation of Generator $1=313.35 \mathrm{MW}$

Total Generation of Generator $2=913.35 \mathrm{MW}$

Total Generation of Generator $7=265.85 \mathrm{MW}$

Total Generation of Generator $13=438.25 \mathrm{MW}$

Total Generation of Generator $15=418.025 \mathrm{MW}$

Total Generation of Generator $16=466.925 \mathrm{MW}$

Total Generation of Generator $18=430.075 \mathrm{MW}$

Total Generation of Generator $21=387.82 \mathrm{MW}$

Total Generation of Generator $22=291.55 \mathrm{MW}$

Total Generation of Generator $23=324.825 \mathrm{MW}$

Therefore, the total Generation cost $=$ INR 1390745.915

The Transmission charges are considered as $10 \%$ of the Generation charges. Therefore, Transmission Charges $=$ $10 \%$ X $1381256.50=$ INR 138125.65. The transmission cost matrix $\square C_{L G} \square$ must be considered such that the

Transmission Charges when evaluated come to approximately $10 \%$ of the Generation Charges. Here, in this case, the loss which has to be contributed by each generated is neglected. So the total amount of active power to be generated and transmitted by each generator to meet 
theload is given by $\square P_{L G} \square$. Therefore the total transmission costis given by $\square P_{L G} \square X \square C_{L G} \square$ which is calculated as shown in Table IV. It is to note that the authors have calculated only the transmission basic charges by RED method.

Therefore, the Total Transmission Cost obtained will be the sum of all the elements of the above matrix = INR133133.9131 $\approx 10 \%$ of the Generation Charges.

It is to note that Table IV gives the total transmission cost by multiplying $\square P_{L G} \square X \square C_{L G} \square \square$ i.e (A generator's share in meeting a load)*(cost/MW in transferring the said share (power) for the distance between their location). Hence, there is no need of any details for Table IV (row /column wise)

TABLE IV. EVALUATION OF TRANSMISSION BASIC CHARGES

\begin{tabular}{|c|c|c|c|c|c|c|c|c|c|c|c|c|c|c|c|c|}
\hline 363.05 & 467.78 & 461.42 & 469.63 & 479.81 & 456.21 & 401.97 & 448.25 & 469.33 & 499.99 & 499.99 & 499.99 & 499.99 & 499.99 & 461.67 & 499.99 & 499.99 \\
\hline 467.76 & 366.91 & 458.40 & 467.26 & 478.23 & 452.74 & 499.99 & 444.15 & 466.90 & 499.99 & 377.00 & 499.99 & 499.99 & 499.99 & 458.66 & 466.49 & 499.99 \\
\hline 461.47 & 58.42 & 73.54 & 60.82 & 73.95 & 443.48 & 499.99 & 433.18 & 371.09 & 499.99 & 499.99 & 499.99 & 439.77 & 467.58 & 450.54 & 199.99 & 499.99 \\
\hline 469.68 & 467.25 & 460.82 & 363.93 & 479.51 & 455.51 & 499.99 & 447.44 & 468.83 & 499.99 & 500.01 & 397.24 & 499.99 & 455.55 & 442.28 & 500.00 & 500.00 \\
\hline 279.85 & 78.21 & 3.95 & 79.49 & 52.80 & 270.4 & 299. & 252.40 & 279.26 & 285.57 & 299.99 & 300.01 & 299 & 299.98 & 74.13 & 39.62 & $\overline{99.99}$ \\
\hline 456.26 & 452.70 & 443.44 & 455.51 & 470.44 & 374.95 & 499.99 & 424.09 & 455.02 & 396.09 & 473.44 & 499.99 & 500.02 & 500.01 & 443.83 & 199.99 & 499.99 \\
\hline 448.39 & 4.18 & 3.22 & 7.49 & 2.45 & 4.18 & 3.64 & 38.27 & 446.89 & 494.60 & 500.03 & 500.04 & 500.04 & 500.05 & 33.66 & 77.35 & 73.89 \\
\hline 469.33 & 466.88 & 71.19 & 68.83 & 79.27 & 455.00 & 499.99 & 446.79 & 364.28 & 499.99 & 499.99 & 499.99 & 429.87 & 464.23 & 460.61 & 9.99 & 99.99 \\
\hline 499.94 & 499.95 & 499.94 & 499.95 & 485.51 & 396.04 & 499.95 & 494.48 & 499.95 & 316.28 & 454.56 & 499.93 & 499.95 & 499.96 & 499.95 & 473.99 & 499.94 \\
\hline 500.00 & 377.02 & 500.00 & 00.00 & 500.00 & 473.42 & 500.00 & 500.00 & 500.00 & 454.61 & 334.53 & 500.00 & 500.00 & 500.00 & 500.00 & 433.02 & 500.00 \\
\hline 500.00 & 500.00 & 500.00 & 397.23 & 00.00 & 500.00 & 500.00 & 500.00 & 500.00 & 500.00 & 500.00 & 366.08 & 500.00 & 411.54 & 441.27 & 500.00 & $\overline{500.00}$ \\
\hline 500.00 & 500.00 & 467.58 & 455.56 & 500.00 & 500.00 & 500.00 & 500.00 & 462.26 & 500.00 & 500.00 & 411.54 & 474.54 & 404.02 & 437.85 & 500.00 & 500.00 \\
\hline 461.71 & 458.65 & 450.53 & 442.29 & 474.12 & 443.83 & 500.00 & 433.61 & 460.63 & 500.00 & 500.00 & 441.27 & 500.00 & 437.85 & 398.92 & 500.00 & 500.00 \\
\hline 499.99 & 446.46 & 499.99 & 499.99 & 439.63 & 499.99 & 499.99 & 477.30 & 499.99 & 474.07 & 474.07 & 499.99 & 499.99 & 499.99 & 499.99 & 362.43 & 499.99 \\
\hline 499.10 & 499.10 & 499.10 & 499.12 & 499.13 & 499.10 & 459.29 & 472.86 & 499.10 & 499.10 & 499.10 & 499.10 & 414.17 & 499.10 & 499.10 & 499.10 & 369.34 \\
\hline
\end{tabular}

\section{IV.CONCLUSION}

In this paper, three transmission network cost allocation methodologies are compared using standard 24 bus RTS. A complete analysis with a comparative study has been made on all the three techniques.Table I provides the transmission cost allocation to generators by $Z_{B U S}$ technique. Table II shows the total transmission cost allocation for all the generators and demands by the first two techniques.From table II, it is inferred that both the above methods allocate most of the costs for using line 23 to generators 21,22 , and 23 . This is because all the generators are electrically close to that line, and their productions are comparatively high.The RED method allocates the transmission charges based on the relative location of load nodes with respect to the generator nodes. This method is conceptually simple and can be implemented using the network configuration and generation/load conditions in a day-to-day operation of power systems. The main advantage of this method lies in its applicability to consider multiple contracts/transactions simultaneously. Comparing the overall transmission cost obtained in all the three techniques, RED method is very accurate in estimating and allocating the transmission cost in the transmission pricing scheme. From the results, it is also found that RED method is very effective in transmission cost allocation.

\section{ACKNOWLEDGMENT}

The authors acknowledge the support provided by the K.vimalakumar ,Assistant professor, J.N.T.U.A.C.E.P, Pulivendula, INDIA for carrying out this work

\section{REFERENCES}

[1] M. Ilic, F. Galiana, and L. Fink,"Power Systems Restructuring: Engineering and Economics" Norwell, MA: Kluwer, 1998.

[2] J. W. M. Lima, "Allocation of transmission fixed rates: An overview," IEEE Trans. Power Syst, vol. 11, no. 3, pp. 1409-1418, Aug. 1996.

[3] J. Bialek, "Topological generation and load distribution factors for supplement charge allocation in transmission open access," IEEE Trans.Power Syst., vol. 12, no. 3, pp. 1185-1193, Aug. 1997

[4] S. Kirschen, R. N. Allan, and G. Strbac, "Contributions of individual Generators to loads and flows," IEEE Trans. Power Syst., vol. 12, no. 1,pp. 52-60, Feb. 1997.

[5] W. Y. Ng, "Generalized generation distribution factors for power system security evaluations," IEEE Trans. Power App. Syst., vol.PAS-100, pp. 1001-1005, Mar. 1981

[6] D. Galiana, A. J. Conejo, and H. A. Gil, "Transmission network cost allocation based on equivalent bilateral exchanges," IEEE Trans. Power Syst., vol. 18, no. 4, pp. 1425-1431, Nov. 2003.

[7] H. A. Gil, F. D. Galiana, and A. J. Conejo, "Multiarea transmission network cost allocation," IEEE Trans. Power Syst., vol. 20, no. 3, pp. 1293-1301, Aug. 2005

[8] J. Conejo, F. D. Galiana, and I. Kockar, "Z-bus loss allocation," IEEE Trans. Power Syst., vol. 16, no. 1, pp. 105-110, Feb. 2001

[9] R. Berger and V. Vittal, Power Systems Analysis, 2nd ed. Englewood Cliffs, NJ: Prentice-Hall, 2000.

[10] Reliability Test System Task Force, "The IEEE reliability test system 1996," IEEE Trans. Power Syst., vol. 14, no. 3, pp. 10101020, Aug. 1999. 